\title{
18F-Fluorodeoxyglucose Uptake in Abdominal Aortic Aneurysms: A Useful Biomarker of AAA Rupture Risk
}

\author{
Kosmas I. Paraskevas, ${ }^{1}$ Dimitri P. Mikhailidis, ${ }^{2}$ and Frank J. Veith ${ }^{3,4}$ \\ ${ }^{1}$ Sheffield Vascular Institute, Northern General Hospital, Sheffield S5 7AU, UK \\ ${ }^{2}$ Department of Clinical Biochemistry (Vascular Disease Prevention Clinics), University College London Medical School, \\ Royal Free Hospital Campus, University College London (UCL), London NW3 2QG, UK \\ ${ }^{3}$ Divisions of Vascular Surgery, New York University Langone Medical Center, New York, NY 10016, USA \\ ${ }^{4}$ Division of Vascular Surgery, The Cleveland Clinic, Cleveland, OH 44106, USA \\ Correspondence should be addressed to Kosmas I. Paraskevas; paraskevask@hotmail.com
}

Received 9 August 2014; Accepted 19 August 2014; Published 18 September 2014

Academic Editor: Thomas Schmitz-Rixen

Copyright (C) 2014 Kosmas I. Paraskevas et al. This is an open access article distributed under the Creative Commons Attribution License, which permits unrestricted use, distribution, and reproduction in any medium, provided the original work is properly cited.

Moris et al. discuss several emerging biomarkers implicated in the pathophysiology of abdominal aortic aneurysms (AAAs) [1]. These include biomarkers related to AAA extracellular matrix homeostasis or proteolysis, cellular or signalling pathways, proteins released by intraluminal thrombi, circulating cells and inflammation, metabolomics and genetic biomarkers. One emerging biomarker which probably also deserves to be mentioned is 18F-fluorodeoxyglucose (18FFDG).

18F-FDG uptake detected by positron emission tomography (PET) is used to assess hypermetabolic activity of cells in tumors and inflammatory processes $[2,3]$. Inflammation plays a key role in the development of AAAs [4]. AAA regions displaying increased 18F-FDG uptake show increased inflammatory activity and are enriched in leukocytes [5]. Increased 18F-FDG uptake in AAAs is associated with inflammation, aortic wall instability, and rupture risk [6]. Therefore, 18F-FDG uptake might be a new technique to study AAA disease in vivo and may improve prediction of AAA rupture risk $[6,7]$. The prognostic value of $18 \mathrm{~F}-$ FDG uptake was verified by a study reporting increased focal uptake of $18 \mathrm{~F}-\mathrm{FDG}$ in patients with large, rapidly expanding, or symptomatic AAAs that are prone to rupture [8].
In a recent study [9], biopsies of the AAA wall were obtained from regions with no 18F-FDG uptake and from regions positive for 18F-FDG uptake, both at the site positive for uptake and at a distant negative site of the AAA wall. The sites with a positive 18F-FDG uptake were characterized by a higher number of adventitial inflammatory cells and by a reduction of smooth muscle cells in the media compared with the negative 18F-FDG samples [9]. It was concluded that positive 18F-FDG uptake in the AAA wall is associated with an active inflammatory process and alterations of the expression of genes involved in the remodelling of adventitia and collagen degradation, which potentially participate in the weakening of the AAA wall preceding rupture [9]. Another use of 18F-FDG may be in endovascular AAA repair (EVAR) procedures [10]. Increased 18F-FDG uptake following EVAR may be an indirect predictor of AAA sac enlargement due to the presence of an endoleak (even if this is not detected by imaging modalities) and increased AAA rupture risk [10].

18F-FDG uptake may be a more promising biomarker for AAAs than some of those discussed by the authors (e.g., cystatin $C$ and neutrophil gelatinase-associated lipocalin [NGAL]) [1]. There is evidence that NGAL and cystatin C are affected by kidney function, as well as by statins [11-13]. In 
turn, patients with AAAs may have impaired kidney function and may well be on statins [14]. To make things even more complicated, statins can improve renal function in vascular patients [15]. These factors make the use of these markers subject to confounding.

In conclusion, 18F-FDG may prove to be a useful biomarker in the pathogenesis of AAAs. Future studies should investigate these possibilities.

\section{Conflict of Interests}

The authors declare that there is no conflict of interests regarding the publication of this paper.

\section{References}

[1] D. Moris, E. Mantonakis, and E. Avgerinos, "Novel biomarkers of abdominal aortic aneurysm disease: identifying gaps and dispelling misperceptions," BioMed Research International, vol. 2014, Article ID 925840, 13 pages, 2014.

[2] M. Miyagawa, R. Yokoyama, Y. Nishiyama, A. Ogimoto, J. Higaki, and T. Mochizuki, "Positron emission tomographycomputed tomography for imaging of inflammatory cardiovascular diseases," Circulation Journal, vol. 78, no. 6, pp. 1302-1310, 2014.

[3] B. Tantiwongkosi, F. Yu, A. Kanard, and F. R. Miller, "Role of (18)F-FDG PET/CT in pre and post treatment evaluation in head and neck carcinoma," World Journal of Radiology, vol. 6, no. 5, pp. 177-191, 2014.

[4] K. I. Paraskevas, M. Andrikopoulou, E. Anastasakis, D. Perrea, and D. P. Mikhailidis, "Oxidative stress in the pathogenesis of abdominal aortic aneurysms: a possible pathway for the effect of statins?" Angiology, vol. 61, no. 2, pp. 226-227, 2010.

[5] O. D. Defawe, R. Hustinx, J. O. Defraigne, R. Limet, and N. Sakalihasan, "Distribution of F-18 fluorodeoxyglucose (F18 FDG) in abdominal aortic aneurysm: high accumulation in macrophages seen on PET imaging and immunohistology," Clinical Nuclear Medicine, vol. 30, no. 5, pp. 340-341, 2005.

[6] C. Reeps, M. Essler, J. Pelisek, S. Seidl, H.-H. Eckstein, and B.-J. Krause, "Increased 18F-fluorodeoxyglucose uptake in abdominal aortic aneurysms in positron emission/computed tomography is associated with inflammation, aortic wall instability, and acute symptoms," Journal of Vascular Surgery, vol. 48, no. 2, pp. 417-423, 2008.

[7] K. I. Paraskevas, A. A. Tzovaras, F. Gentimi, Z. S. Kyriakides, and D. P. Mikhailidis, "Predictors of abdominal aortic aneurysm (AAA) growth and AAA rupture risk besides AAA Size: fact or fiction?” Angiology, vol. 61, no. 4, pp. 321-323, 2010.

[8] N. Sakalihasan, H. Van Damme, P. Gomez et al., "Positron emission tomography (PET) evaluation of abdominal aortic aneurysm (AAA)," European Journal of Vascular and Endovascular Surgery, vol. 23, no. 5, pp. 431-436, 2002.

[9] A. Courtois, B. V. Nusgens, R. Hustinx et al., " ${ }^{18}$ F-FDG uptake assessed by PET/CT in abdominal aortic aneurysms is associated with cellular and molecular alterations prefacing wall deterioration and rupture," Journal of Nuclear Medicine, vol. 54, no. 10, pp. 1740-1747, 2013.

[10] K. I. Paraskevas, A. A. Tzovaras, V. Stathopoulos, F. Gentimi, and D. P. Mikhailidis, "Increased fluorodeoxyglucose uptake following endovascular abdominal aortic aneurysm repair: a predictor of endoleak?" The Open Cardiovascular Medicine Journal, vol. 4, pp. 117-119, 2010.

[11] S. M. Alharazy, N. Kong, R. Saidin et al., "Serum neutrophil gelatinase-associated lipocalin and cystatin $\mathrm{C}$ are early biomarkers of contrast-induced nephropathy after coronary angiography in patients with chronic kidney disease," Angiology, vol. 65, no. 5, pp. 436-442, 2014.

[12] R. G. Fassett, I. K. Robertson, M. J. Ball, D. P. Geraghty, J. W. Cardinal, and J. S. Coombes, "Effects of atorvastatin on NGAL and cystatin $\mathrm{C}$ in chronic kidney disease: a post hoc analysis of the LORD trial," Nephrology Dialysis Transplantation, vol. 27, no. 1, pp. 182-189, 2012.

[13] S. M. Alharazy, N. Kong, R. Saidin et al., "Neutrophil gelatinaseassociated lipocalin as an early marker of contrast-induced nephropathy after coronary angiography," Angiology, vol. 65, no. 3, pp. 216-223, 2014.

[14] K. I. Paraskevas, A. S. Wierzbicki, and D. P. Mikhailidis, "Statins and noncardiac vascular disease," Current Opinion in Cardiology, vol. 27, no. 4, pp. 392-397, 2012.

[15] M. E. Alnaeb, F. Youssef, D. P. Mikhailidis, and G. Hamilton, "Short-term lipid-lowering treatment with atorvastatin improves renal function but not renal blood flow indices in patients with peripheral arterial disease," Angiology, vol. 57, no. 1, pp. 65-71, 2006. 


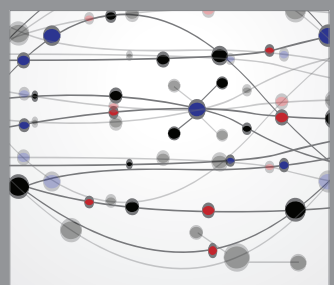

The Scientific World Journal
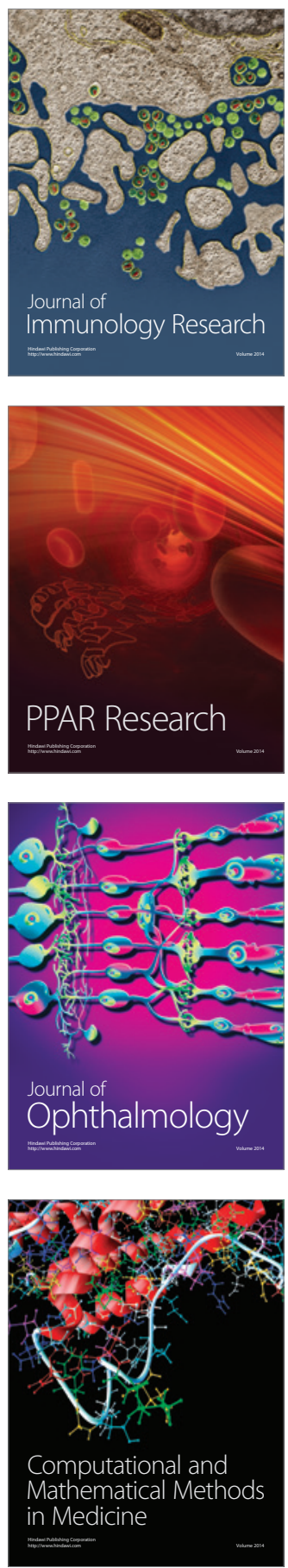

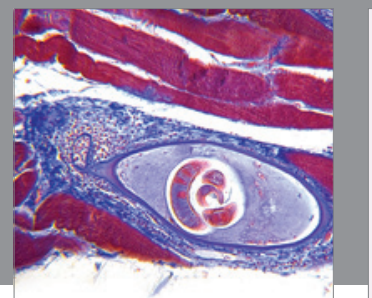

Gastroenterology

Research and Practice
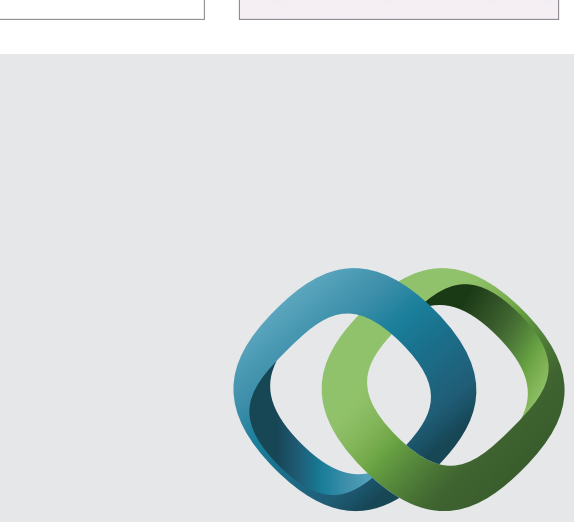

\section{Hindawi}

Submit your manuscripts at

http://www.hindawi.com
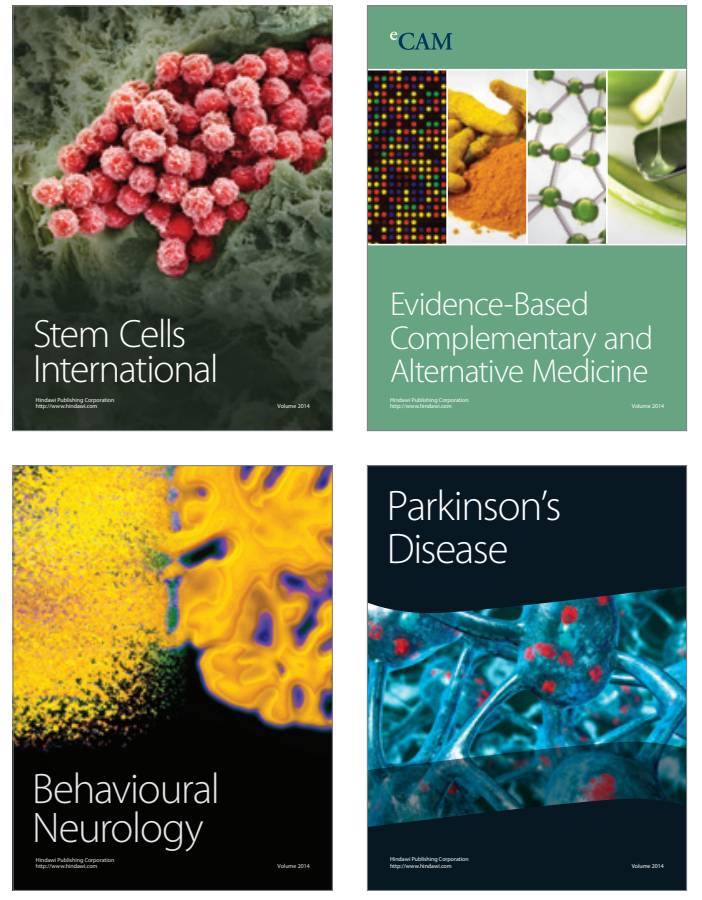
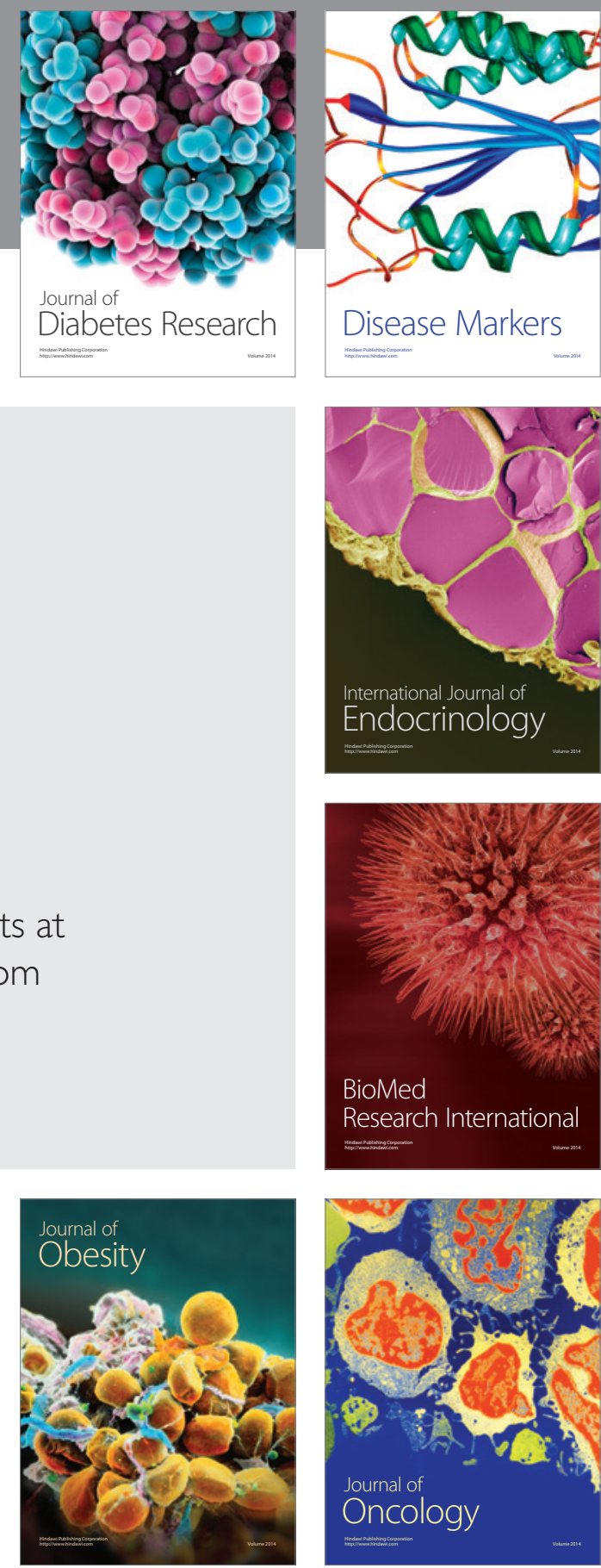

Disease Markers
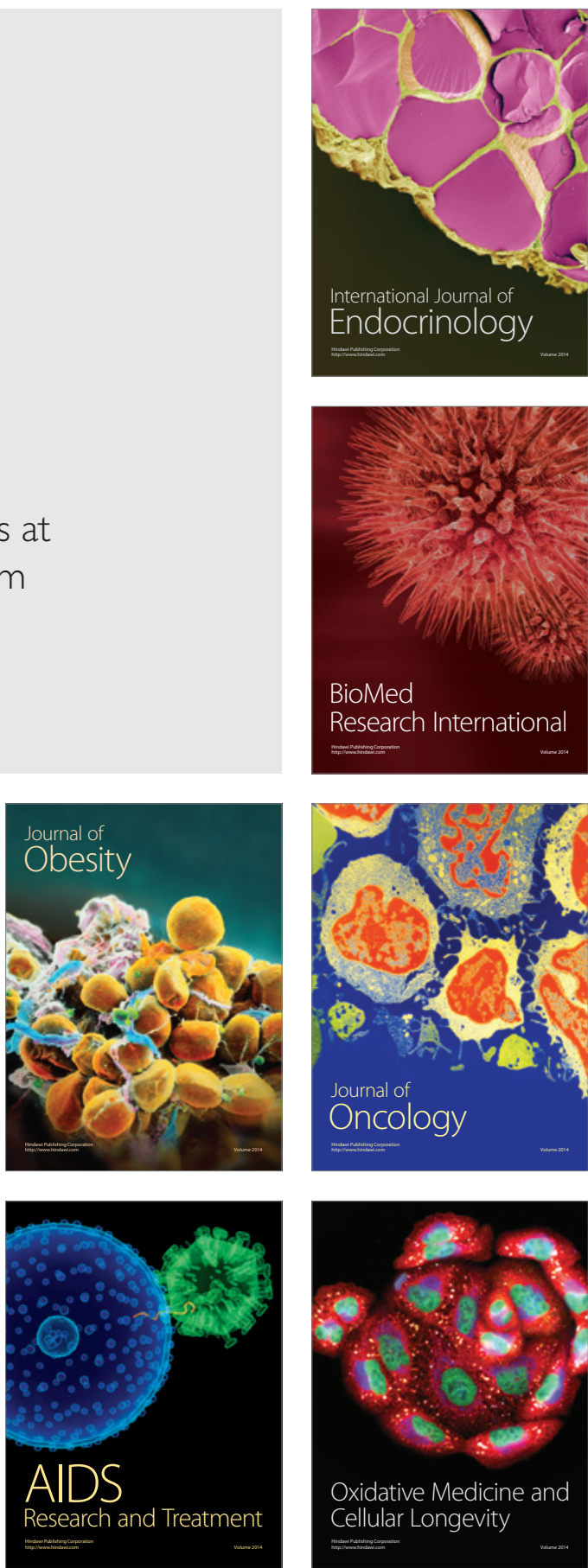